\title{
Calcium gluconate alleviates the toxic effect of hydrofluoric acid on human dermal fibroblasts through the Wnt/ $\beta$-catenin pathway
}

\author{
JINXIA PENG ${ }^{1}$, RUI LIU ${ }^{2}$, LING PENG ${ }^{3}$ and HONGTAO JIA ${ }^{4}$ \\ ${ }^{1}$ Department of Burn and Plastic Surgery, Shiyan People's Hospital, People's Hospital Affiliated to \\ Hubei University of Medicine; ${ }^{2}$ Department of Oncology, Taihe Hospital Affiliated to Hubei University of Medicine; \\ ${ }^{3}$ Department of Health Management, People's Hospital of Shiyan Economic Development Zone; ${ }^{4}$ Department of \\ Urological Surgery, Shiyan People's Hospital, People's Hospital Affiliated to Hubei University of Medicine, \\ Shiyan, Hubei 442000, P.R. China
}

Received November 3, 2017; Accepted May 15, 2018

DOI: $10.3892 / \mathrm{ol} .2018 .8975$

\begin{abstract}
The present study was performed to determine the molecular mechanism of calcium gluconate $(\mathrm{CG})$ in alleviating the toxic effect of hydrofluoric (HF) acid on human dermal fibroblasts (HDFs). HDF morphology was observed by optical microscopy and the vimentin immunofluorescence assay. Cell viability and apoptosis were evaluated by the Cell Counting Kit-8 and Annexin V/propidium iodide assays, respectively. The levels of apoptosis-associated factors, as well as Wnt2, Wnt3a and $\beta$-catenin were detected by reverse transcription-quantitative polymerase chain reaction and western blotting. Levels of matrix metalloproteinase (MMP)-1 and basic fibroblast growth factor (bFGF) were detected by ELISA and western blotting. Carboxyterminal propeptide of type I collagen (CICP) was detected by ELISA, while L-Hydroxyproline (L-HYP) was detected by colorimetry. First, the morphology of normal HDFs was observed. Cell viability was inhibited and apoptosis was increased in a doseand time-dependent manner following treatment with HF acid $[0,2,4,6,8,10$ and $20 \%(\mathrm{v} / \mathrm{v})]$ for $0,2,4,6,8,10$ and $20 \mathrm{~min}$. The effects were blocked by CG at different doses $(50,100$ and $200 \mu \mathrm{mol} / \mathrm{l})$ and time points $(6,12$ and $24 \mathrm{~h})$, following treatment with $8 \%(\mathrm{v} / \mathrm{v}) \mathrm{HF}$ acid for $6 \mathrm{~min}$. The levels of Caspase-3, B-cell lymphoma (Bcl)-2 associated X protein, Wnt2, Wnt3a and $\beta$-catenin were decreased, whereas Bcl-2 was increased by CG treatment dose-dependently, when compared with HF control. CG promoted the expression of MMP-1, bFGF and L-HYP, and inhibited CICP, when compared with HF control. Based on the present results, CG alleviated the toxic effect of
\end{abstract}

Correspondence to: Dr Hongtao Jia, Department of Urological Surgery, Shiyan People's Hospital, People's Hospital Affiliated to Hubei University of Medicine, 39 Chaoyangzhong Road, Shiyan, Hubei 442000, P.R. China

E-mail: hongtaojia46@163.com

Key words: calcium gluconate, hydrofluoric acid, human dermal fibroblasts, apoptosis, wound healing
HF acid on HDFs by regulating the Wnt/ $\beta$-catenin signaling pathway.

\section{Introduction}

Hydrofluoric (HF) acid is a highly dangerous toxic substance, widely used in industrial engineering. Exposure could result in unique severe cutaneous burns, systemic toxicity, and occasionally lethal toxicity. HF acid burns are notorious for progressive tissue necrosis and severe pain, which can penetrate the skin and destruct deep tissue layers, as well as the bone. In tissues, $\mathrm{HF}$ acid can react with calcium and magnesium ions, producing insoluble fluoride salts which can induce myocardium defects, hypocalcemia and changes in levels of active substances. High concentrations or prolonged exposure may cause death (1). As low as $2.5 \% \mathrm{HF}$ acid has been reported to cause skin burns and even induce death.

Numerous topical and parenteral treatments have been developed and are currently available. For example, calcium salts have been proven effective for systemic dermal injuries. Calcium gluconate (CG), aloe gel, A + D ointment, magnesium ointment and Zephiran (benzalkonium chloride) are applied topically, and their efficacy has been extensively investigated. Of these, topical CG gel has emerged as an effective treatment of HF acid burns, with recovery signs becoming apparent soon after treatment initiation. Previous studies found that topical applications and subcutaneous injections of CG could significantly deter the damage caused by burns in the first $24 \mathrm{~h}$, as well as enhance tissue recovery as thetreatment progresses. It has been reported that $\mathrm{CG}$ can react with $\mathrm{HF}$ acid to form insoluble, non-toxic calcium fluoride. However, little is known regarding the molecular mechanism underlying its therapeutic effect.

Wound healing involves the repairing of tissue defects through enhanced cellular proliferation, migration and differentiation. Human dermal fibroblasts (HDFs) are critical cells for wound healing and tissue repairing after skin injuries $(2,3)$. HDFs are responsible for generating connective tissue in the dermis and repairing skin lesions. Moreover, HDFs produce proteins, such as laminin and fibronectin, which can aid the formation of extracellular matrix between dermis and 
epidermis, effectively combining epidermal cells and, thus, forming a new top layer of the skin $(4,5)$. Therefore, HDFs are essential for proper recovery following skin injury (6-8).

In this study, we investigated the possible roles of CG in attenuating the toxic effect of HF acid on HDFs. We studied the CG-driven activation of the wound healing process, by assessing HDF proliferation, collagen synthesis and migratory properties. The obtained results may offer new insights into the use of CG for the treatment of dermal lesions.

\section{Materials and methods}

Cell culture. HDFs were purchased from ATCC (Guangzhou, China) and cultured in DMEM medium (Gibco; Thermo Fisher Scientific, Inc., Waltham, MA, USA) containing $10 \%$ fetal bovine serum (FBS; Gibco; Thermo Fisher Scientific, Inc.) and $1 \%$ penicillin/streptomycin (Invitrogen; Thermo Fisher Scientific, Inc.) at $37^{\circ} \mathrm{C}$, in a $5 \% \mathrm{CO}_{2}$ incubator. Cells of logarithm phase were used in our study. Cell morphology of HDFs was observed by an optical microscope (Olympus, Tokyo, Japan).

Vimentin immunofluorescence staining. Cell morphology of HDFs was evaluated by vimentin immunofluorescence staining assay. $\mathrm{HDF}$ cells were inoculated on sterile coverslips in 6-well plates and cultured for 2 days at $37^{\circ} \mathrm{C}$ in a $5 \% \mathrm{CO}_{2}$ incubator. Paraformaldehyde functioned on HDFs and membranes were broken then. HDFs were probed with rabbit anti-human vimentin primary antibody and then with the appropriate FITC-conjugated donkey anti-rabbit secondary antibody. After that, HDFs were immunofluorescence stained by 4',6-diamidino-2-phenylindole (DAPI). Finally, images were obtained by DM5000 epifluorescence microscope (Leica, Wetzlar, Germany).

Cell viability assay. The effect of HF acid on cell viability of HDFs was evaluated by the Cell Counting Kit-8 (CCK8) assay. HDFs were treated with HF acid of different concentrations $[0,2,4,6,8,10$ and $20 \%(\mathrm{v} / \mathrm{v})]$, for determined amounts of time $(0,2,4,6,8,10$ and $20 \mathrm{~min})$. Briefly, following each treatment, cells were seeded in 96-well plates, at an initial density of $5 \times 10^{3}$ cells/well, and incubated for the indicated amounts of time. Subsequently, $20 \mu \mathrm{l}$ CCK- 8 reagent were added into each well. The plates were then incubated for another $1 \mathrm{~h}$. The optical density (OD) values were read at $450 \mathrm{~nm}$, using a microplate reader (Thermo Fisher Scientific, Inc.). Data were expressed as percentage of viable cells as follows: Relative viability $(\%)=\left[\mathrm{A}_{450}\right.$ (treated) $-\mathrm{A}_{450}$ (blank) $] /\left[\mathrm{A}_{450}\left(\right.\right.$ control) $-\mathrm{A}_{450}$ (blank)] x100\%.

The effect of CG on cell viability of HF-acid-injured HDFs was also evaluated by the CCK8 assay. HDFs were pre-treated with CG at different concentrations (50, 100 and $200 \mu \mathrm{mol} / \mathrm{l})$ for 6,12 and $24 \mathrm{~h}$, respectively, after which they were treated with $8 \%(\mathrm{v} / \mathrm{v}) \mathrm{HF}$ acid for $6 \mathrm{~min}$. The results were compared with cell viability of HDFs solely treated with $8 \%(\mathrm{v} / \mathrm{v}) \mathrm{HF}$ acid for $6 \mathrm{~min}$ (HF control). The detection was performed as described above.

Apoptosis detection. The apoptosis status was measured by Annexin V/PI double-stain assay, according to the
Table I. Primers used in reverse transcription-quantitative polymerase chain reaction analysis.

\begin{tabular}{|c|c|c|}
\hline Name & Type & Sequence $\left(5^{\prime}-3^{\prime}\right)$ \\
\hline \multirow[t]{2}{*}{ Caspase-3 } & Forward & $\begin{array}{l}\text { TGTGAGGCGGTTGTAGAAG } \\
\text { AGT }\end{array}$ \\
\hline & Reverse & $\begin{array}{l}\text { CACACCCACCGAAAACCA } \\
\text { GAG }\end{array}$ \\
\hline \multirow[t]{2}{*}{ Bax } & Forward & TGCTTCAGGGTTTCATCCA \\
\hline & Reverse & GGCCTTGAGCACCAGTTT \\
\hline \multirow[t]{2}{*}{$\mathrm{Bcl}-2$} & Forward & $\begin{array}{l}\text { ACGGTGGTGGAGGAGCT } \\
\text { CTT }\end{array}$ \\
\hline & Reverse & CGGTTGACGCTCTCCACAC \\
\hline \multirow[t]{2}{*}{ Wnt2 } & Forward & $\begin{array}{l}\text { CTGACCTGATGCAGACGC } \\
\text { AAG }\end{array}$ \\
\hline & Reverse & $\begin{array}{l}\text { AGGAGCCACCTGTAGCTCT } \\
\text { CATGTA }\end{array}$ \\
\hline \multirow[t]{2}{*}{ Wnt3a } & Forward & $\begin{array}{l}\text { GATGGTGTCTCGGGAGT } \\
\text { TCG }\end{array}$ \\
\hline & Reverse & CCGTGGCACTTGCACTTGA \\
\hline \multirow[t]{2}{*}{$\beta$-catenin } & Forward & $\begin{array}{l}\text { ATAAGAGCTCCTTGTGC } \\
\text { GGC }\end{array}$ \\
\hline & Reverse & $\begin{array}{l}\text { GGCCATGTCCAACTCCA } \\
\text { TCA }\end{array}$ \\
\hline \multirow[t]{2}{*}{ GAPDH } & Forward & $\begin{array}{l}\text { TGACTTCAACAGCGACAC } \\
\text { CCA }\end{array}$ \\
\hline & Reverse & $\begin{array}{l}\text { CACCCTGTTGCTGTAGCC } \\
\text { AAA }\end{array}$ \\
\hline
\end{tabular}

Bcl-2, B-cell lymphoma 2; Bax, Bcl-2 assocaited X protein.

manufacturer's protocol (Biovision, Mountain View, CA, USA). Briefly, after HF acid treatment or additional CG pre-treatment, both floating and trypsinized adherent cells $\left(5 \times 10^{5}\right)$ were collected and resuspended in $500 \mu \mathrm{l}$ binding buffer containing $5 \mu \mathrm{l}$ Annexin V fluorescein isothiocyanate and additional $5 \mu \mathrm{l}$ Propidium Iodide (PI; Invitrogen; Thermo Fisher Scientific, Inc.). Then cells were incubated for $5 \mathrm{~min}$ in the dark, at the room temperature. Analysis was immediately performed with a flow cytometer (BD Biosciences, Franklin Lakes, NJ, USA). Cell Quest software was used to analyze the apoptosis rate.

Reverse transcription-quantitative polymerase chain reaction $(R T-q P C R)$. The mRNA expression levels were measured by RT-qPCR. Total RNA was extracted from HDFs with different treatment respectively, using RNeasy kit (Qiagen, Valencia, CA, USA), and cDNA was reversely transcribed with $1 \mu \mathrm{g}$ RNA using a kit of Quantiscript Reverse Transcriptase (Qiagen), according to the protocol provided by the manufacture. PCR amplification was performed for $30 \mathrm{sec}$ at $95^{\circ} \mathrm{C}$, followed by 40 cycles: Denaturation at $95^{\circ} \mathrm{C}$ for $15 \mathrm{sec}$, annealing/extension at $60^{\circ} \mathrm{C}$ for $30 \mathrm{sec}$ in ABI 7300 Thermocycler (Applied Biosystems; Thermo Fisher Scientific, Inc.) using Fast SYBR Green Master Mix (Applied Biosystems; Thermo Fisher Scientific, Inc.). The oligo nucleotide primer sequences were displayed in Table I. 
A

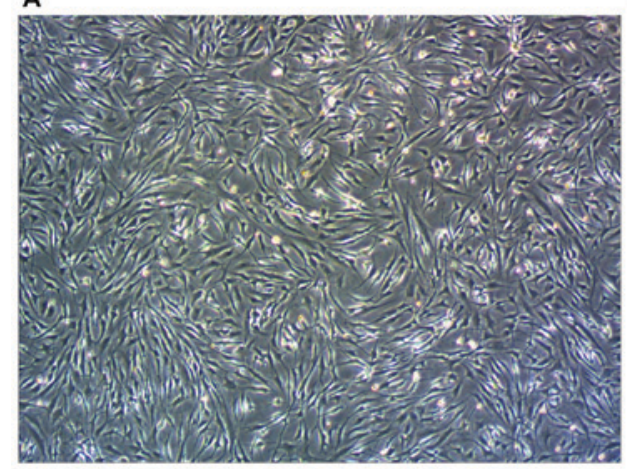

C

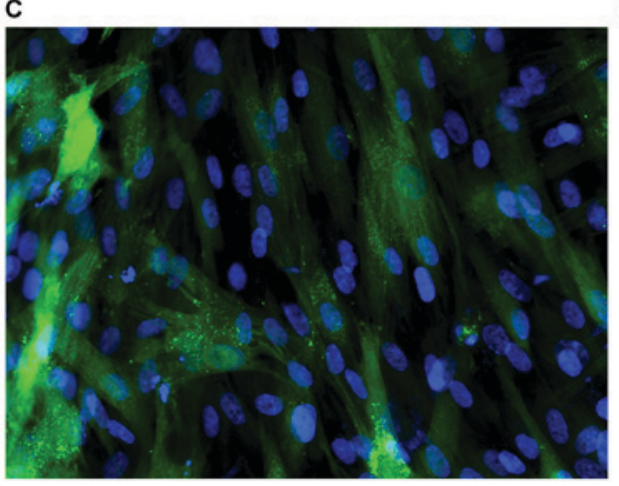

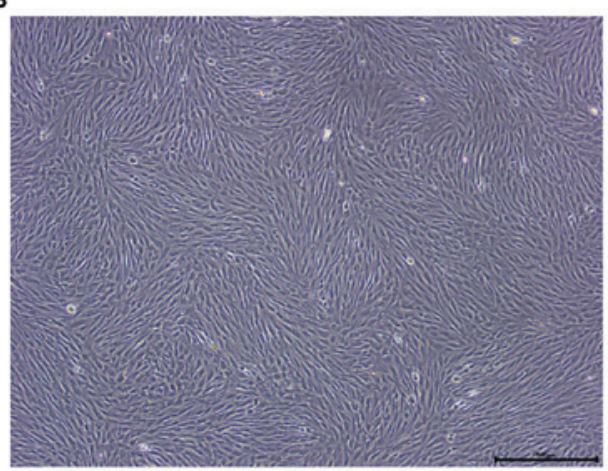

D

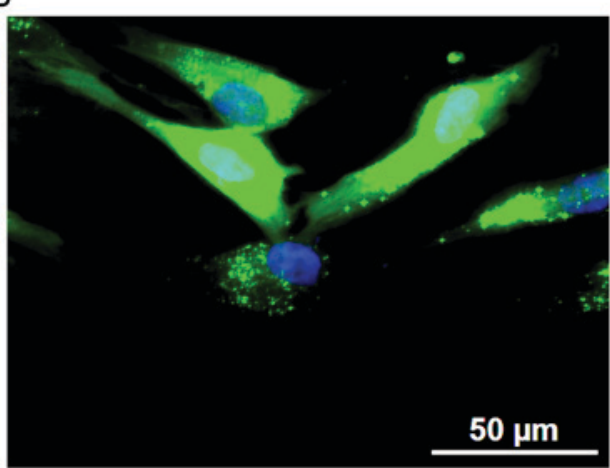

Figure 1. Optical and fluorescence micrographs of cell morphologies of HDFs. (A and B) Cell morphologies of HDFs were detected by optical microscope. (C and D) Cell morphologies of HDFs were detected by vimentin immunofluorescence staining assay (magnification, x100 and x200). HDFs, human dermal fibroblasts.

Western blot analysis. The concentrations of proteins were determined by BCA assay (Beyotime, Nantong, China). Then proteins were subjected to sodium dodecyl sulfate-polyacrylamide gel electrophoresis (SDS-PAGE) and electroblotted onto a polyvinylidenefluoride(PVDF)membrane (Amersham, Amersham, UK). Following the blockage of 5\% nonfat dry milk for $1 \mathrm{~h}$, the blotting membranes were probed with the primary antibodies at $4^{\circ} \mathrm{C}$ overnight respectively. which were then probed with appropriate HRP-conjugated secondary antibodies. The PVDF membranes were exposed to X-ray film, and the immunoreactive bands were detected by reaction with enhanced chemiluminescense (ECL) detection system reagents (Amersham, Arlington Heights, IL, USA). For loading control, the membrane was probed with a monoclonal antibody for glyceraldehyde-3-phosphate dehydrogenase (GAPDH). Lab Works Image Acquisition and Analysis Software (UVP, Upland, CA, USA) were used to quantify band intensities. Antibodies were purchased from Abcam (Cambridge, UK).

Enzyme-linked immunosorbent assay (ELISA). The amounts of proteins were determined by an ELISA kit (R\&D Systems, Minneapolis, MN, USA), following the manufacturer's instructions. Samples and standard substances were added into 96-well plates. After incubation for $90 \mathrm{~min}$ at $37^{\circ} \mathrm{C}$, biotinylated antibodies were added into the wells and incubated for another $60 \mathrm{~min}$ at $37^{\circ} \mathrm{C}$. After being rinsed for 3 times, Avidin peroxidase complex was added and the plates were incubated for $30 \mathrm{~min}$. Next, after being rinsed for 3 times, tetramethylbenzidine coloring reagent was added and incubated for $15 \mathrm{~min}$ at $37^{\circ} \mathrm{C}$. Finally, the OD values were read at $450 \mathrm{~nm}$ by a microplate reader (Thermo Fisher Scientific, Inc.). Protein amounts were calculated by the generated standard curve.

Colorimetric assay. The level of L-hydroxyproline (L-HYP) in HDFs was determined by the colorimetric method. In this assay, L-HYP was oxidized by chloramine $\mathrm{T}$, and redundant chloramine $\mathrm{T}$ was removed by perchloric acid. After that, dimethyl amino benzaldehyde was added to form a red complex. OD values were detected at $558 \mathrm{~nm}$, by an ultraviolet spectrophotometer (Mettler Toledo, Greifensee, Switzerland). The amount of L-HYP in each sample was calculated using the obtained standard curve.

Statistical analysis. All results were expressed as mean \pm standard deviations of three independent experiments. Statistical analysis was performed using a SPSS 13.0 statistical package (SPSS, Inc., Chicago, IL, USA) and data were subjected to one-way analysis of variance (ANOVA), followed by Dunnett's test. $\mathrm{P}<0.05$ was considered significant; $\mathrm{P}<0.01$ was considered especially significant.

\section{Results}

HDF morphology. HDF morphology was detected by optical microscopy and the vimentin immunofluorescence staining assay. When observed under an optical microscope, HDF cells appeared oval or applanate-star-like, with plump cytoplasm, and clear nucleolus, consistent with the classical morphology of HDFs. When the number of cells increased, HDFs arranged closely with decreased cell space, and became spiral- or fence-shaped (Fig. 1A and B). Following the microscopic 

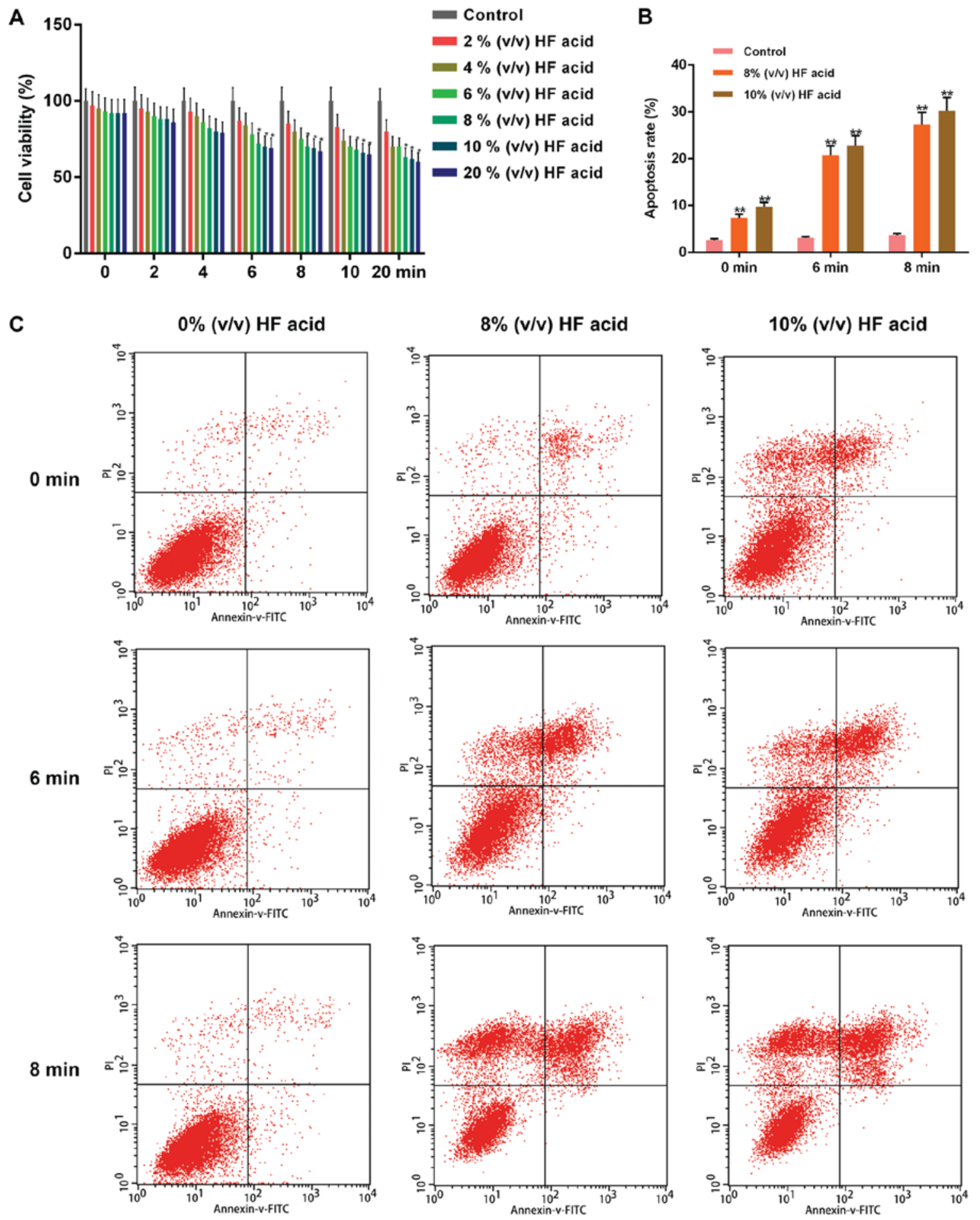

Figure 2. Analysis of cell viability and apoptosis of HDFs treated with HF. (A) Cell viability of HDFs were detected by Cell Counting Kit- 8 assay when treated with $\mathrm{HF}$ acid of different concentrations $[0,2,4,6,8,10$ and $20 \%(\mathrm{v} / \mathrm{v})]$ for determined times $(0,2,4,6,8,10$ and 20 min), in a dose- and time-dependent manners. (B) Apoptosis of HDFs was promoted when treated with HF acid of different concentrations $[0,2,4,6,8,10$ and $20 \%(\mathrm{v} / \mathrm{v})]$ for specific times $(0,2$, 4, 6, 8, 10 and $20 \mathrm{~min}$ ) in a dose- and time-dependent manners, as determined by (C) flow cytometry. Data were presented as the mean \pm standard deviation ( $\mathrm{n}=6$ /group). ${ }^{*} \mathrm{P}<0.05$ and ${ }^{* *} \mathrm{P}<0.01$ vs. control. HDFs, human dermal fibroblasts; HF, hydrofluoric; FITC, fluorescein isothiocyanate; PI, propidium iodide.

assessment, HDF morphology was detected by the vimentin immunofluorescence staining assay. As a member of the intermediate filament protein family, vimentin is the main component of the cytoskeleton, being expressed in cells derived from mesoblastema, especially HDFs. Consequently, HDFs can be detected by intracellular vimentin in the cytoplasm. Results showed that the oval cell nucleus was dyed blue by DAPI, whereas the spindle- or applanate-star-shaped cytoplasm was dyed green, specifically indicating the presence of vimentin around the nucleus (Fig. 1C and D).

Analysis of cell viability and apoptosis of HDFs treated with HF acid. The effect of HF acid on the viability of HDFs, measured by the CCK8 assay, was shown in Fig. 2A. 
A

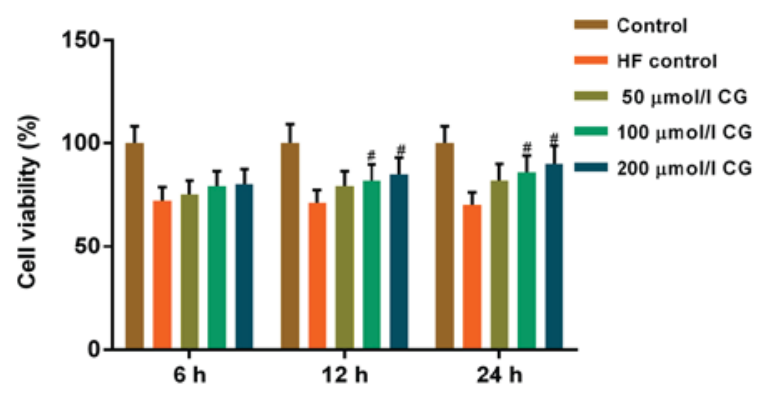

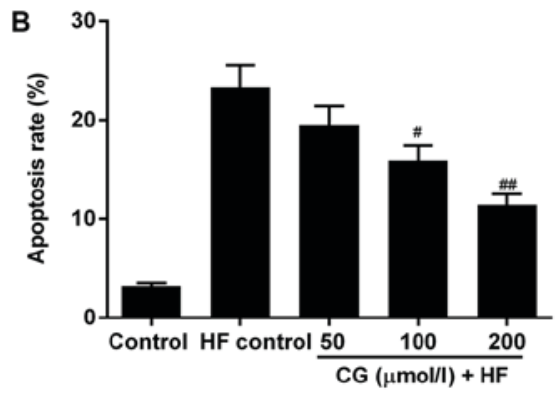
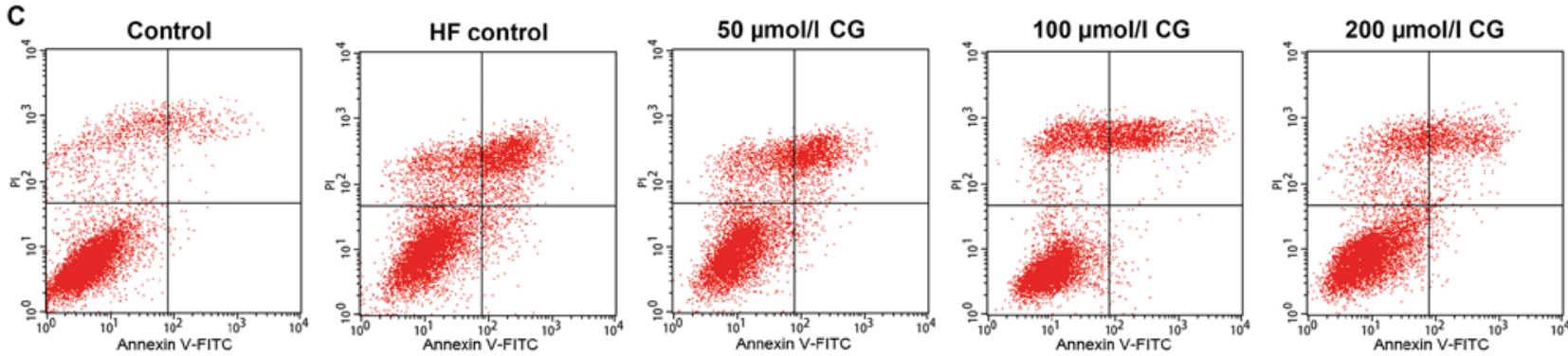

D

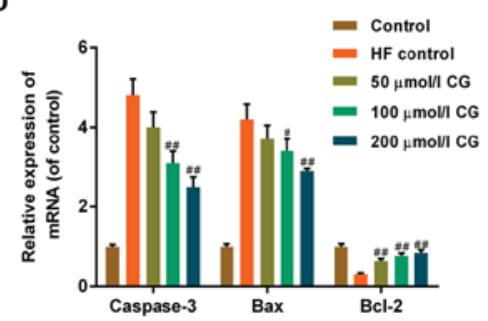

E

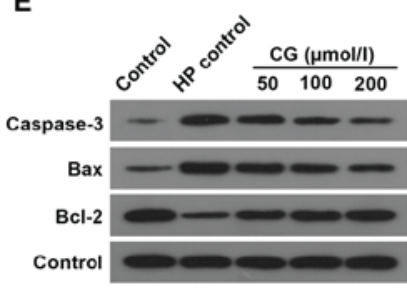

$\mathbf{F}$

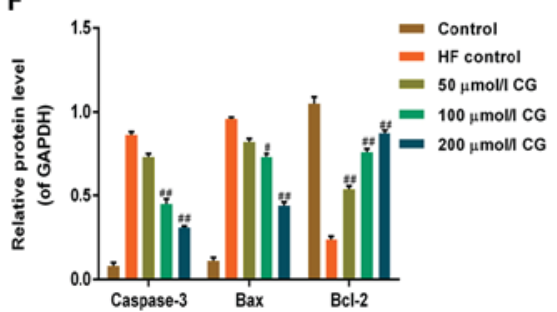

Figure 3. Function of CG on cell viability and apoptosis of HDFs treated with HF. (A) Compared with HDFs treated with HF acid (HF control), the cell viability of HDFs was elevated when pre-treated with CG of different concentrations (50, 100 and $200 \mu \mathrm{mol} / \mathrm{l})$ for 6,12 or $24 \mathrm{~h}$ respectively, then treated with $8 \%$ (v/v) HF acid for $6 \mathrm{~min}$, in a dose- and time-dependent manners, as determined by Cell Counting Kit-8 assay. (B) Compared with HDFs treated with HF acid, apoptosis of HDFs was reduced when pre-treated with CG of different concentrations (50, 100 and $200 \mu \mathrm{mol} / 1)$ for 6,12 or $24 \mathrm{~h}$ respectively, then treated with $8 \%$ (v/v) HF acid for 6 min, in a dose- and time-dependent manner, (C) as determined by flow cytometry. (D) The mRNA expression levels of Caspase-3 and Bax were decreased, and the levels of Bcl-2 increased following CG treatment, in dose-dependent manner, when compared with HF control. (E and F) The protein levels of Caspase-3 and Bax were decreased, and the levels of Bcl-2 increased following CG treatment, in dose-dependent manner when compared with HF control. Data were presented as mean \pm standard deviation ( $\mathrm{n}=6 /$ group). ${ }^{\#} \mathrm{P}<0.05$ and ${ }^{\# \#} \mathrm{P}<0.01$ vs. HF control. HDFs, human dermal fibroblasts; HF, hydrofluoric; CG, calcium gluconate; Bcl-2, B-cell lymphoma 2; Bax, Bcl-2-assocaited X protein; FITC, fluorescein isothiocyanate; PI, propidium iodide.

Cell viability was suppressed in the HF-treated groups in dose-dependent $(2,4,6,8,10$ and $20 \%(\mathrm{v} / \mathrm{v}))$ and time-dependent manners $(2,4,6,8,10$ and $20 \mathrm{~min})$, compared with the control group. HDF viability varied significantly when treated with 8,10 and $20 \%(\mathrm{v} / \mathrm{v}) \mathrm{HF}$ acid for $6,8,10$ or 20 min $(\mathrm{P}<0.05)$, compared with the control group. However, the viability of HDFs treated with $20 \%$ (v/v) HF acid for 10 or 20 min was similar to that of cells treated with 8 or $10 \%$ (v/v) HF acid for 6 or $8 \mathrm{~min}$, so detection of apoptosis was performed on HDFs treated with lower concentrations of HF acid $[8$ and $10 \%(\mathrm{v} / \mathrm{v})]$ for 6 and $8 \mathrm{~min}$.

We performed Annexin V/PI double-staining to quantify cellular apoptosis, and used flow cytometry for analysis (Fig. 2B and C). Apoptosis increased as the level of phosphatidylserine in the outer leaflet of the plasma membrane increased, being detected by Annexin $\mathrm{V}$ binding to the surface of the cells. The apoptotic rate of HF-acid-treated HDFs increased significantly, about 3 -fold of control. The treatment of $8 \%(\mathrm{v} / \mathrm{v})$ $\mathrm{HF}$ acid for 6 min was subjected to subsequent investigations.

Function of CG on cell viability, apoptosis status and apoptosis-related factors of HDFs treated with HF acid.
The effect of CG on the viability of HF-acid-injured HDFs was measured by the CCK8 assay, and the results were shown in Fig. 3A. Cell viability was suppressed in $8 \%(\mathrm{v} / \mathrm{v})$ HF-acid-treated HDFs (treatment duration: $6 \mathrm{~min}$ ) without CG pre-treatment. When HDFs were pre-treated with $\mathrm{CG}$, cell viability increased in dose-dependent (50, 100 and $200 \mu \mathrm{mol} / \mathrm{l})$ and time-dependent (6, 12 and $24 \mathrm{~h}$ ) manners, compared with $\mathrm{HF}$ control. The statistical variation was considered significant when cells were treated with 100 or $200 \mu \mathrm{mol} / \mathrm{l} \mathrm{CG}$ for 12 or $24 \mathrm{~h}$. CG pre-treatment with different concentrations $(50,100$, $200 \mu \mathrm{mol} / \mathrm{l}$ ) for $24 \mathrm{~h}$ was chosen to assess apoptosis, as well as for the following detections. We performed Annexin V/PI double-staining to quantify apoptosis, and used flow cytometry for the analysis (Fig. 3B and C). The apoptotic rates of HDFs decreased when pre-treated with $\mathrm{CG}$ for $24 \mathrm{~h}$, prior to the 6 min-treatment with $8 \%(\mathrm{v} / \mathrm{v}) \mathrm{HF}$ acid, in a dose-dependent (50, 100 and $200 \mu \mathrm{mol} / \mathrm{l})$ manner.

RT-qPCR and western blotting were performed to detect mRNA and protein expression levels of important apoptosis-related factors, such as Caspase-3, Bcl-2 associated $\mathrm{X}$ protein (Bax) and B-cell lymphoma (Bcl)-2 (Fig. 3D-F). The mRNA and protein levels of Caspase-3 and Bax 
A

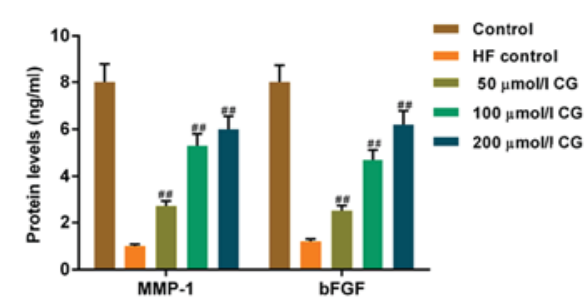

D

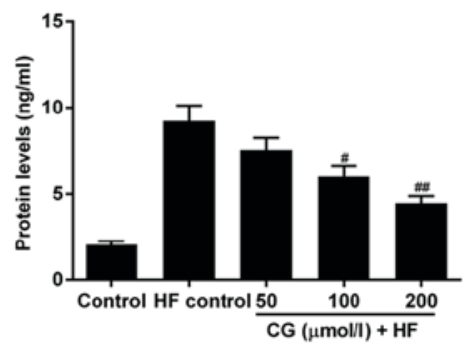

B

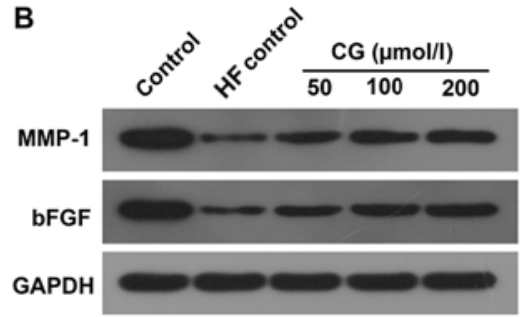

$\mathbf{F}$

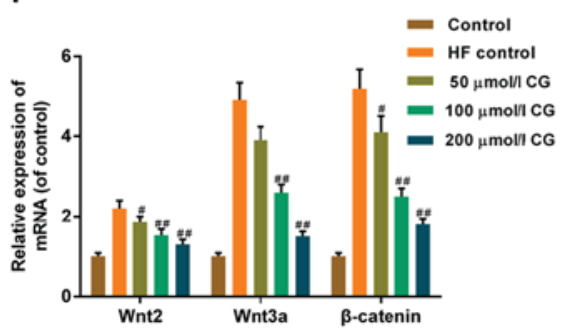

G

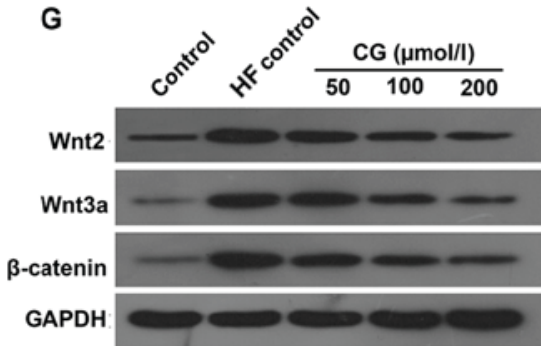

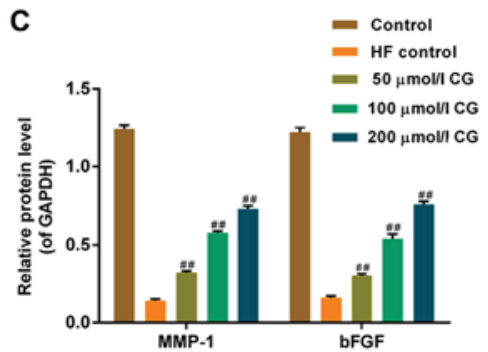

E

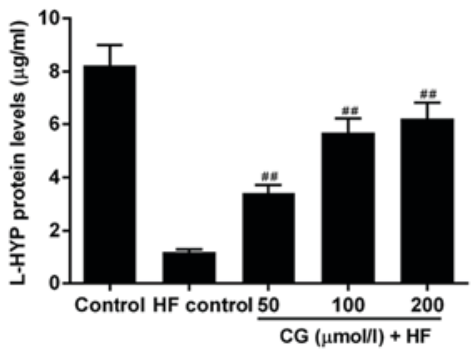

H

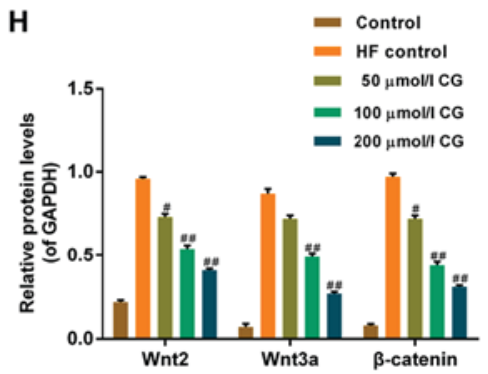

Figure 4. Function of CG on cell division and fiber hyperplasia associated factors in HDFs treated with HF. (A) ELISA assay revealed that when pre-treated with CG, protein levels of MMP-1 and bFGF increased in a dose-dependent manner when compared with HF control. (B) Western blot confirmed that, (C) when pre-treated with CG, the protein levels of MMP-1 and bFGF increased in a dose-dependent manner when compared with HF control. (D) ELISA assay showed that protein levels of carboxyterminal propeptide of type I collagen in CG pre-treated HDFs decreased in a dose-dependent manner, compared with HF control. (E) Colorimetric method demonstrated that protein levels of L-HYP increased in a dose-dependent manner compared with HF control. (F) Reverse transcription-quantitative polymerase chain reaction showed that mRNA expression levels of Wnt2, Wnt $3 \mathrm{a}$ and $\beta$-catenin decreased in a dose-dependent manner compared with HF control. (G) Western blot showed that (H) protein levels of Wnt2, Wnt $3 \mathrm{a}$ and $\beta$-catenin decreased in a dose-dependent manner when compared with HF control. Data were presented as mean \pm standard deviation ( $=6 /$ group). ${ }^{\#} \mathrm{P}<0.05$ and ${ }^{\# \#} \mathrm{P}<0.01 \mathrm{vs}$. HF control. HDFs, human dermal fibroblasts; HF, hydrofluoric; CG, calcium gluconate; MMP, matrix metalloproteinase; bFGF, basic fibroblast growth factor; L-HYP, L-Hydroxyproline.

increased, whereas the level of Bcl-2 decreased significantly in HF-acid-treated cells, compared with control. When pre-treated with CG, the mRNA and protein levels of Caspase-3 and Bax decreased, and the level of Bcl-2 was restored in a dose-dependent manner, compared with HF control.

Function of CG on cell division and fiber-hyperplasia-related factors of HDFs treated with HF acid. The critical repair function of CG on HF-acid-induced cell viability suppression and apoptosis promotion in HDFs, prompted us to further explore the potential underlying mechanism by measuring the relevant genes and proteins.

ELISA and Western blot assays showed that, protein levels of matrix metalloproteinase (MMP)-1 and basic fibroblast growth factor (bFGF) decreased significantly in HF control, compared with the control group. When pre-treated with CG, protein levels of MMP-1 and bFGF were restored in a dose-dependent manner, compared with HF control (Fig. 4A-C). ELISA was performed to detect the level of secretory protein carboxyterminal propeptide of type I collagen (CICP) (Fig. 4D). The level of CICP increased remarkably in HF control, compared with the control group. When pre-treated with CG, the protein level of CICP decreased in a dose-dependent manner, compared with HF control. As detected by the colorimetric method, the protein level of L-HYP decreased significantly in HF control, compared with the control group. When pre-treated with CG, the level of L-HYP was restored in a dose-dependent manner, compared with HF control (Fig. 4E).

RT-PCR and Western-blotting were performed to detect the mRNA and protein expressions of important factors in the Wnt pathway, such as Wnt2, Wnt3a and $\beta$-catenin (Fig. 4F-H). The mRNA and protein levels of Wnt2, Wnt3a and $\beta$-catenin increased notably in HF control, compared with the control group. When pre-treated with CG, the mRNA and protein levels of Wnt2, Wnt $3 \mathrm{a}$ and $\beta$-catenin decreased in a dose-dependent manner, compared with HF control.

\section{Discussion}

HF acid is a highly dangerous, caustic, inorganic acid, which can cause severe cutaneous burns, systemic toxicity and occasionally lethal toxicity. CG has been proven to be 
a more effective therapy for dermal and systemic injuries. Nevertheless, the underlying molecular mechanism is yet to be elucidated.

The primary objective of this study was to elucidate the major molecular mechanism behind the restorative effect of $\mathrm{CG}$ on HF-acid-injured HDFs. In the present study, the classical morphology of HDFs was observed under an optical microscope, visible as oval or applanate-star-shaped, with plump cytoplasm and clear nucleolus; as the cellular density increased HDFs became spiral- or fence-shaped. Vimentin is a member of the intermediate filament protein family. As the main component of the cytoskeleton, it is expressed in cells derived from mesoblastema, particularly HDFs. Therefore, we further assessed the morphology of HDFs by detecting the presence of vimentin around the nucleus, using an immunofluorescent green dye.

$\mathrm{HF}$ acid burns are notorious for causing progressive tissue necrosis and severe pain. Apoptosis is a process of programmed cell death that occurs in multicellular organisms, important in maintaining the metabolic balance. As an important component of granulation tissue, new vessels play key roles in wound healing, providing the necessary nutrients for the migration and proliferation of HDFs.

Our study showed that CG could promote cell viability and decrease apoptotic rates of HDFs, which are destroyed by HF acid resulting in skin injury, and thus support wound healing. Furthermore, we studied the expression variations of critical factors in cell proliferation and apoptosis, such as Caspase-3, Bcl-2 and Bax, showing consistent changes. Caspase- 3 is an important member of the caspase family (cysteinyl aspartate specific proteinases), as a conjunct activating factor in apoptosis signal transduction, which directly participates in cell regulation, signal transduction and late apoptosis. Bcl-2 activates the caspase pathway, facilitates cell mitosis and inhibits apoptosis by dissolving protein structures, resulting in tumor proliferation (9). In contrast, Bax can facilitate apoptosis by forming a heterodimer with Bcl-2, essentially deterring its activity. When pre-treated with $\mathrm{CG}, \mathrm{mRNA}$ and protein levels of Caspase- 3 and Bax decreased, whereas thelevel of $\mathrm{Bcl}-2$ increased in dose-dependent manners, compared with HF-acid-injured HDFs. This suggests that the restorative function of CG on HF-acid-induced cell viability suppression and apoptosis promotion of HDFs is related to the molecular mechanism responsible for apoptosis.

$\mathrm{HF}$ acid can penetrate the skin and destruct deep tissue layers, as well as the bone. Bone metabolic disorder is considered a common phenomenon in fluorosis and the main characteristic of fluorosis of the bone. CICP, the carboxyl terminal cut from type I collagen (Col-I) before fiber formation, is the characteristic of Col-I and bone development, for Col-I is the main collagen of the bone scaffold (10). In addition, L-HYP is considered the characteristic amino acid of collagen. Being the main residue of collagen proteins degradation, the content of L-HYP is constant, accounting for more than $10 \%$ of collagen amino acids. Therefore, L-HYP levels could reflect collagen synthesis and HDF status. MMP-1, secreted by HDFs, is a critical enzyme regulating collagen degradation, especially Col-I (11). Self-renewal and damage repair of skin tissues are related to the metabolic proliferation and directional differentiation of epidermal stem cells. Cytokines like bFGF, a kind of heparin-combined polypeptide mitogen, are critical factorspromoting cell division and proliferation, while regulating cell differentiation and immunology. Previous studies have even identified a connection between bFGF and wound healing (11). Our study showed that CG could inhibit the expression of CICP and promote the expression of L-HYP, MMP-1 and bFGF, as these were increased (CICP) and decreased (L-HYP, MMP-1 and bFGF), respectively, in HF-acid-treated HDFs. This strongly suggests that CG could influence cell division or fiber hyperplasia. Therefore, $\mathrm{CG}$ may attenuate the toxic effect of HF acid on HDFs by regulating the expression of cell division or fiber hyperplasia-related factors.

Wnt was first discovered in mouse breast cancer epithelial cells. Though most research on the Wnt pathway is concentrated on the regulation of stem cell differentiation, organ development and tumorigenesis (12), the Wnt pathway also participates in wound healing after skin injury, progression of fibrotic diseases and activation of fibrotic effector cells. The related specific function and molecular mechanism may depend on the type of tissue or organ. Research on the involvement of the Wnt pathway in wound healing and fibrosis has been increasing in recent years. Labus et al (13) and Okuse et al (14) found that the Wnt pathway is activated in the early stage of wound healing, and Konigshoff et al discovered activated Wnt pathway in pulmonary fibrosis $(15,16)$. There is crosstalk between the Wnt pathway and the TGF- $\beta / \mathrm{Smad}$ pathway, as $\beta$-catenin is a scaffold protein linking the cytoplasmic tail of classical cadherins of the endothelium to the actin cytoskeleton in the Wnt downstream pathway, and its down regulation can give rise to tumor metastasis and fibrotic diseases $(17,18)$. Wnt2 and Wnt3a both induce abundant cellular changes in several key signaling molecules, including $\beta$-catenin, in the canonical $\mathrm{Wnt} / \beta$-catenin pathway. Our study showed that CG could inhibit the expression of Wnt 2 , Wnt3a and $\beta$-catenin, which were increased in the HF-acid-treated HDFs. This indicates that CG attenuated the toxic effect of $\mathrm{HF}$ acid on HDFs by regulating the Wnt/ $\beta$-catenin pathway. However, as Caspase-3, Bax and Bcl-2, as well as Wnt2, Wnt3a and $\beta$-catenin are classical pathways that can be affected by many factors, it may be that more specific mechanisms are involved. Similarly, there are specific inhibitors for both the Wnt pathway and cell apoptosis. We will perform more blocking experiments to assess the specificity of these effects and pathways, in a future study.

Collectively, these data suggest that $\mathrm{CG}$ attenuated the toxic effect of HF acid on HDFs by promoting cell viability, while inhibiting apoptosis and related factors. Moreover, CG may regulate cell division or fiber hyperplasia-related factors, and also the $\mathrm{Wnt} / \beta$-catenin pathway. These findings provide novel evidence in favor of utilizing $\mathrm{CG}$ in the treatment of HF-acid-induced wounds.

\section{Acknowledgements}

Not applicable.

\section{Funding}

No funding was received. 


\section{Availability of data and materials}

The analyzed data sets generated during the study are available from the corresponding author on reasonable request.

\section{Authors' contributions}

JP designed the research and treated cells. RL, LP and HJ performed the other experiments and drafted the manuscript. All authors have read and approved the final manuscript.

\section{Ethics approval and consent to participate}

Not applicable.

\section{Consent for publication}

Not applicable.

\section{Competing interests}

The authors declare that they have no competing interests.

\section{References}

1. Hojer J, Personne M, Hulten P and Ludwigs U: Topical treatments for hydrofluoric acid burns: A blind controlled experimental study. J Toxicol Clin Toxicol 40: 861-866, 2002.

2. Shamis Y, Hewitt KJ, Carlson MW, Margvelashvilli M, Dong S, Kuo CK, Daheron L, Egles C and Garlick JA: Fibroblasts derived from human embryonic stem cells direct development and repair of 3D human skin equivalents. Stem Cell Res Ther 2: 10, 2011.

3. Andriani F, Margulis A, Lin N, Griffey S and Garlick JA: Analysis of microenvironmental factors contributing to basement membrane assembly and normalized epidermal phenotype. J Invest Dermatol 120: 923-931, 2003.

4. Smola H, Thiekötter G and Fusenig NE: Mutual induction of growth factor gene expression by epidermal-dermal cell interaction. J Cell Biol 122: 417-429, 1993.

5. Chmielowiec J, Borowiak M, Morkel M, Stradal T, Munz B, Werner S, Wehland J, Birchmeier C and Birchmeier W: c-Met is essential for wound healing in the skin. J Cell Biol 177: 151-162, 2007.

6. Matsumoto $\mathrm{K}$ and Nakamura T: Hepatocyte growth factor (HGF) as a tissue organizer for organogenesis and regeneration. Biochem Biophys Res Commun 239: 639-644, 1997.

7. Sorrell JM and Caplan AI: Fibroblast heterogeneity: More than skin deep. J Cell Sci 117: 667-675, 2004.
8. Sorrell JM, Baber MA and Caplan AI: Clonal characterization of fibroblasts in the superficial layer of the adult human dermis. Cell Tissue Res 327: 499-510, 2007.

9. Rashed L, Gharib DM, Hussein RE, Tork O and Abusree A: Combined effect of bone marrow derived mesenchymal stem cells and nitric oxide inducer on injured gastric mucosa in a rat model. Tissue Cell 48: 644-652, 2016.

10. Scalinci SZ, Scorolli L, Meduri A, Grenga PL, Corradetti G and Metrangolo C: Effect of basic fibroblast growth factor and cytochrome c peroxidase combination in transgenic mice corneal epithelial healing process after excimer laser photoablation. Clin Ophthalmol 5: 215-221, 2011.

11. Gallego-Munoz P, Ibares-Frias L, Valsero-Blanco MC, Cantalapiedra-Rodriguez R, Merayo-Lloves $J$ and Martinez-Garcia MC: Effects of TGF $\beta 1$, PDGF-BB, and bFGF, on human corneal fibroblasts proliferation and differentiation during stromal repair. Cytokine 96: 94-101, 2017.

12. Sokol SY: Maintaining embryonic stem cell pluripotency with Wnt signaling. Development 138: 4341-4350, 2011.

13. Labus MB, Stirk CM, Thompson WD and Melvin WT: Expression of Wnt genes in early wound healing. Wound Repair Regen 6: 58-64, 1998.

14. Okuse T, Chiba T, Katsuumi I and Imai K: Differential expression and localization of WNTs in an animal model of skin wound healing. Wound Repair Regen 13: 491-497, 2005.

15. Konigshoff M, Balsara N, Pfaff EM, Kramer M, Chrobak I, Seeger $\mathrm{W}$ and Eickelberg O: Functional Wnt signaling is increased in idiopathic pulmonary fibrosis. PLoS One 3: e2142, 2008.

16. Konigshoff M, Kramer M, Balsara N, Wilhelm J, Amarie OV, Jahn A, Rose F, Fink L, Seeger W, Schaefer L, et al: WNT1-inducible signaling protein-1 mediates pulmonary fibrosis in mice and is upregulated in humans with idiopathic pulmonary fibrosis. J Clin Invest 119: 772-787, 2009.

17. Cheon S, Poon R, Yu C, Khoury M, Shenker R, Fish J and Alman BA: Prolonged beta-catenin stabilization and tcf-dependent transcriptional activation in hyperplastic cutaneous wounds. Lab Invest 85: 416-425, 2005.

18. Dômont J, Salas S, Lacroix L, Brouste V, Saulnier P, Terrier P, Ranchère D, Neuville A, Leroux A, Guillou L, et al: High frequency of beta-catenin heterozygous mutations in extra-abdominal fibromatosis: A potential molecular tool for disease management. Br J Cancer 102: 1032-1036, 2010.

(i) $($ This work is licensed under a Creative Commons (c) NO NO Attribution-NonCommercial-NoDerivatives 4.0 International (CC BY-NC-ND 4.0) License. 